\title{
Integrando saberes con los estudiantes en la comunidad Bajo de Afuera del cantón Mon- tecristi
}

\section{Integrating knowledge with students in the Bajo de Afuera community of the Montecristi canton}

\section{Integrando saberes con los estudiantes.}

\author{
Raquel Vera Velázquez ${ }^{(\mathbf{1})}$ \\ Kirenia Maldonado Zúñiga ${ }^{(2)}$ \\ Alfredo Lesvel Castro Landin ${ }^{(3)}$ \\ Wilfrido Javier Del Valle Holguín ${ }^{(4)}$
}

(1) Universidad Estatal del Sur de Manabí. Jipijapa. Ecuador. email: raquelita2015vera@gmail.com ORCID:https://orcid.org/0000-0002-5071-7523

(2)Universidad Estatal del Sur de Manabí Jipijapa. Ecuador. email : kmzmaldonado@gmail.com ORCID:https://orcid.org/0000-0002-3764-5633

(3) Universidad Estatal del Sur de Manabí. Jipijapa. Ecuador. email : alfredolandin@ unesum.edu.ec ORCID: https://orcid.org/0000-0001-6340-8749

(4) Universidad Estatal del Sur de Manabí. Jipijapa. Ecuador. email: wilfrido.del.valle@ uneum.edu.ec ORCID: https://orcid.org/0000-0002-1911-0790

Recibido: 15-10-2020

Aprobado: 6-12-2020
Contacto: raquelita2015vera@gmail.com

\section{Resumen}

El presente trabajo tiene por objetivo conocer las propiedades del suelo de la comunidad Bajo de Afuera del cantón Montecristi, además de comprender su población, sus cultivos, y los principales problemas que lo afectan, conocer las plagas que generalmente en la etapa de cosecha son los causantes de la pérdida del producto y por ende afecta a la economía de los agricultores. El método utilizado fue exploratorio documental a través del estudio e investigación bibliográfica, con la utilización de técnicas como encuestas y entrevistas, contando con la documentación de la parroquia y la experiencia de los pobladores de la comunidad. Con este estudio se fortalecen los conocimientos para lograr un cambio sistémico (de actitud) de las percepciones y criterios, a través de lograr el desarrollo de un proyecto integrador de saberes, que integre los diferentes campos del saber impartidos de una manera natural y según como acontece realmente en la producción de los sistemas agropecuarios con el objetivo de desarrollar, fortificar y motivar las capacidades cognoscitivas, psicomotrices y actitudinales en los estudiantes de la carrera Agropecuaria. El problema de fondo es el escaso entendimiento del ser humano sobre el suelo y sus propiedades por estas razones nos dimos a la tarea de investigar mediante los proyectos integradores de saberes las situaciones del suelo agrícola de las parroquias de la zona 4 provincia de Manabí, para ayudar a la comunidad manabita en la capacitación y estudio del suelo y que logren un mejor resultado en sus cosechas.

Palabras claves: Saber, propiedades, suelo, integración, investigación y recursos. 


\section{Summary}

This research work is based on the identification of the soil properties of the Bajo de Afuera community of the Montecristi canton, in addition to knowing its population, its crops, and the main problems that affect it, knowing the pests that generally in the stage harvest are the cause of the loss of the product and therefore affects the economy of farmers. The method used was exploratory documentary through bibliographic study and research, using techniques such as surveys and interviews, with documentation from the parish and the experience of the community's residents. With this study, knowledge is strengthened to achieve a systemic change (of attitude) of perceptions and criteria, through the development of an integrative project of knowledge, which integrates the different fields of knowledge taught in a natural way and according to how It really happens in the production of the agricultural systems with the objective of developing, fortifying and motivating the cognitive, psychomotor and attitudinal capacities in the students of the Agricultural career. The underlying problem is the poor understanding of the human being about the soil and its properties. For these reasons, we took on the task of investigating the situations of agricultural soil in the parishes of zone 4, Manabí province, through the integrative projects of knowledge to the Manabí community in the training and study of the soil and that they achieve a better result in their harvests.

Key words: Knowledge, properties, land, integration, research and resources.

\section{Introducción}

Las actividades productivas en todo el Ecuador tienen una relación directa con los recursos naturales, entre ellos el suelo, el clima, sus componentes bióticos, sus componentes abióticos; sin dejar de mencionar fenómenos naturales como sismos, erupciones, deslaves, entre otros, que intervienen en las actividades productivas dentro del territorio.

Uno de los problemas a los que se enfrenta la campiña manabita es la degradación de los suelos destinados para fines agrícolas, debido en su gran mayoría de veces por procedimientos inadecuados en el uso de prácticas tradicionales y el avance de la frontera agrícola sobre terrenos de vocación forestal en un área de recarga hídrica.

El suelo es un sistema dinámico que consta de tres fases (sólida, líquida y gaseosa) cuyas propiedades físicas, químicas y biológicas interactúan entre sí para mantener un equilibrio adecuado para el desarrollo de las plantas y su entorno (Tamhane, Motiramani, Bali, y Donahue 1986).

El desarrollo de Proyectos Integradores de Saberes (PIS) en la Universidad Estatal de Manabí y en específico en la carrera agropecuaria es una actividad fundamental dentro del rediseño curricular y de gran desafío, porque implica la integración de diferentes ciencias del saber a través de un proyecto conjunto y coordinado, en este sentido se desarrolló esta propuesta, con los objetivos de desarrollar, fortalecer, motivar las capacidades cognoscitivas, psicomotrices y actitudinales en los estudiantes, a través del fortalecimiento de sus conocimientos en cuanto a la identificación de propiedades del suelo en la zona de planificación 4" (G.M.003-06, 2017).

El Proyecto Integrador de Saberes que se desarrolla en el primer nivel de la carrera Agropecuaria se relaciona con problemas de la realidad, identificados en las labores previas de vinculación con la sociedad, practicas pre-profesionales y en los estudios de documentos normativos.

Según (Valdez, 2014), refiere que el Proyecto Integrador de Saberes (PIS), es la formulación de actividades interrelacionadas de manera planificada y organizada para alcanzar los objetivos previamente determinados con la aplicación de los conocimientos, habilidades y destrezas, así como, el empleo de herramientas y técnicas que permita la viabilidad de los procesos en un tiempo determinado y dentro de los límites establecidos en el presupuesto para cambiar una costumbre humana o dar una solución a un problema.

En la investigación se utilizó el método exploratorio y documental, y se basa en la búsqueda de información secundaria en bibliotecas, centros documentales de instituciones como él (MAGAP, 2013); (ESPAC, 2013); los Gobiernos Autónomos Descentralizados (GADs, 2015) provinciales, municipales y parroquiales, etc. Tiene el propósito de buscar información que integre las asignaturas básicas de primer nivel. (G.M. 003-06, 2017)

En el Proyecto Integrador del Saber se seleccionó el tema "Identificación de propiedades del suelo agrícola de la comunidad bajo de afuera del cantón Montecristi" ya que es afín con la carrera de Agropecuaria y gracias a esto los estudiantes podrán conocer que está relacionada con las ciencias básicas como la Química, Biología, Física y Matemáticas. Por ende, los estudiantes utilizan las capacidades cognoscitivas, psicomotrices y actitudinales 
para el desarrollo del presente proyecto. A través de esta investigación se realiza un estudio de la población de la comunidad Bajo de Afuera, las propiedades del suelo, sus principales cultivos, además de identificar las principales problemáticas que se presentan en cuanto al mismo.

Por otra parte el proyecto integrador de saberes de la carrera Agropecuaria tiene como objetivo conocer el espacio físico, la situación poblacional, geográfica, y la Identificación de Propiedades del Suelo Agrícola en la zona 4 Comunidad Bajo de Afuera perteneciente al cantón Montecristi, donde se lleva a cabo las etapas de diagnóstico, diseño y evaluación, los cuales directamente utilizan los conocimientos de las ciencias básicas (Lógica Matemática, Algebra, Física, Química General, Biología Celular y Expresión Oral y Escrita).

A través de la investigación se motiva a los estudiantes para desarrollar el proyecto y desarrollar las capacidades cognoscitivas, psicomotrices y actitudinales en los estudiantes de la carrera Agropecuaria, a través del estudio sobre "La identificación de propiedades del suelo en la Comuna Bajo de Afuera del Cantón Montecristi”.

El presente trabajo tiene por objetivo conocer las propiedades del suelo de la comunidad Bajo de Afuera del cantón Montecristi, además de comprender su población, sus cultivos, y los principales problemas que lo afectan, conocer las plagas que generalmente en la etapa de cosecha son los causantes de la pérdida del producto y por ende afecta a la economía de los agricultores.

\section{Materiales y Métodos}

La investigación se realizó en el escenario del campo, lo que permitió obtener información precisa, y de esta manera poder identificar las propiedades del suelo agrícola de la comunidad Bajo de Afuera del cantón Montecristi. Se utilizó el método de la investigación exploratoria que impulsa a determinar un mejor diseño de la investigación, recogida de datos y la selección de temas.

Método Exploratorio: Permite sustentar las bases del presente trabajo de investigación a través de información de libros, artículos de revistas, páginas de internet, tomando en cuenta las diferentes definiciones de autores, y realizar un análisis de las mismas, enriqueciendo nuestro trabajo.

Método Estadístico: Se analiza e interpretan los resultados de las técnicas de investigación sobre el tema propuesto de forma cuantitativa y cualitativa.
Para la realización de la presente investigación se aplicaron las siguientes técnicas:

Observación: Esta técnica permitió conocer y adquirir información acerca de la comunidad Bajo de Afuera, sus cultivos, problemáticas en el cultivo, entre otras.

Encuestas: Técnica que permitió dirigirse a los habitantes de la comunidad Bajo de Afuera del cantón Montecristi, para adquirir información acerca del cuidado y protección de las áreas verdes del mencionado lugar. Se realizó una entrevistas al presidente de la comunidad Bajo de Afuera del cantón Montecristi.

\section{Resultados}

El trabajo de investigación está delimitado para la zona 4. En lo referente a la zona de planificación 4Pacífico, se encuentra ubicada al oeste del Ecuador y comprende las provincias de Manabí y Santo Domingo de los Tsáchilas. Limita al norte con la Zona 1-provincia de Esmeraldas y Zona 2 - provincia de Pichincha; al sur con la Zona 5 - provincias de Guayas y Santa Elena; al este con la Zona 5 provincia del Guayas y zona 3 - provincia de Cotopaxi; y al oeste con el océano Pacífico.

En lo concierne al poblado de Bajo de la Palma y Bajo del Pechiche, tiene una extensión de 2782.000 $\mathrm{m}^{2}$ y una población de 4136 habitantes como se muestra en la tabla \# 1. La comunidad cuenta con un solo barrio llamado San José y las calles principales: 18 de septiembre, Targelia Anchundia, Montecristi, Simón Bolívar y Eugenio Espejo.

\begin{tabular}{|l|l|l|l|}
\hline Personas & Edades & $\begin{array}{l}\mathrm{N}^{\circ} \text { de Habi- } \\
\text { tantes }\end{array}$ & $\%$ \\
\hline Hombres & $35-100$ & 1134 & 27 \\
\hline Mujeres & $35-100$ & 1680 & 41 \\
\hline Jóvenes & dic-35 & 912 & 22 \\
\hline Niños & 1 -dic & 410 & 10 \\
\hline Total & & 4136 & 100 \\
\hline
\end{tabular}

Tabla 1. Población comunidad Bajo de Afuera perteneciente al cantón Montecristi.

En lo referente al número de habitantes de la comunidad Bajo de Afuera del cantón Montecristi, se aprecia que prevalece la población femenina, en segunda lugar los hombre, y los jóvenes en el tercer lugar, gráfico 1. 


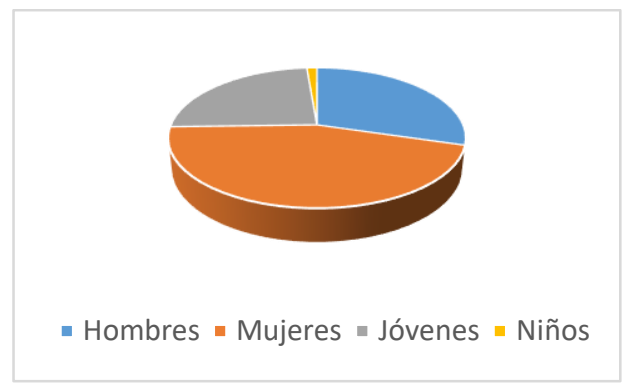

Gráfico 1. Número de habitantes de la comunidad Bajo de Afuera del cantón Montecristi.

Se pudo determinar en el estudio que el agua con la que cuenta la comunidad El Bajo de Afuera, es un pozo de aproximadamente $100 \mathrm{~m}$ de profundidad donde se filtra el agua y de esta manera se abastece al pueblo, a través de conexiones de mangueras por cada casa.

En lo relativo a las cadenas de valor del sector avícola vinculan a los actores y actividades relacionadas con la entrega de productos avícolas, ya sea para consumo propio o para el consumidor final. A esta actividad solo se dedica un 5\% de la población.

Dentro de los resultados se pudo constatar que los habitantes se dedican al cultivo de maíz, al criadero de chivos de castilla y al criadero de aves de corral y que los cultivos agrícolas son afectados en su mayoría por la plaga del gusano cogollero, la plaga de la quemazón y la plaga de cinta amarilla lo que ocasiona una gran pérdida en el sector económico y productivo de la Comuna Bajo de Afuera del cantón Montecristi.

En la parte ganadera, los pobladores se dedican a la crianza de chivos de castilla el $30 \%$ de los habitantes. Y el $15 \%$ personas están dedicadas a la construcción de muebles, para esto utilizan madera de excelente calidad como el guayacán, algarrobo, lo cual les permite ser reconocidos en el mercado, siendo visitados por propios y extraños. A la artesanía están dedicadas el 8\%, y por otra parte el $12 \%$ de las personas son propietarios de la fábrica de ladrillos, cada una de ellas cuenta con 5 empleados, dando un total de 80 personas que se dedican a esta actividad equivalente al $2 \%$. Una parte de la población encuentra empleo dentro del lugar, mientras que el $43 \%$ tienen que salir a trabajar fuera, alrededor de 600 personas salen a laborar en las empresas atuneras.
En lo referente a las propiedades químicas del suelo de la comuna Bajo de Afuera del cantón Montecristi, la reacción de un suelo hace referencia al grado de acidez o basicidad del mismo y generalmente se expresa por medio de un valor de $\mathrm{pH}$ del sistema suelo-agua. El pH es la medida de la concentración de iones de hidrógeno $[\mathrm{H}+]$. Según este valor, un suelo puede ser ácido, neutro o alcalino.

Las propiedades físicas, químicas y biológicas del suelo están influenciadas por la acidez o basicidad del medio, que a su vez condicionan el uso agronómico del suelo. Así, la mayoría de las plantas prefieren rangos de $\mathrm{pH}$ de 5.5 a 7.5, pero algunas especies prefieren suelos ácidos o alcalinos. Sin embargo, cada planta necesita un rango específico de $\mathrm{pH}$, en el que poder expresar mejor su potencialidad de crecimiento.

El análisis de las propiedades físicas del suelo de la comuna el Bajo de Afuera del cantón Montecristi, la condición física de un suelo determina su capacidad de sostenimiento, facilidad para la penetración de raíces, circulación del aire, capacidad de almacenamiento de agua, drenaje, retención de nutrientes, entre otros factores.

En las tablas Nro. 2 y Nro. 3, se presenta las propiedades químicas del suelo agrícola de la comunidad

\begin{tabular}{|l|l|ll|l|l|}
\hline F & M & Ph & Ce & $\begin{array}{l}\text { Tempera- } \\
\text { tura }\end{array}$ \\
\hline F1 & M1 & 7,42 & Básico & $170,1 \mathrm{us} / \mathrm{cm}$ & $21,4^{\circ} \mathrm{C}$ \\
\hline F1 & M2 & 8,75 & L.Alc. & 224,2 us $/ \mathrm{cm}$ & $22,7^{\circ} \mathrm{C}$ \\
\hline F1 & M3 & 8,13 & Básico & $308 \mathrm{us} / \mathrm{cm}$ & $22,3^{\circ} \mathrm{C}$ \\
\hline F1 & M4 & $\begin{array}{l}8,44 \\
\text { no }\end{array}$ & Alcali- & 197,2 us $/ \mathrm{cm}$ & $22,6^{\circ} \mathrm{C}$ \\
\hline
\end{tabular}

Bajo de Afuera del cantón Montecristi.

Tabla 2. Propiedades químicas del suelo de la comunidad Bajo de Afuera del cantón Montecristi.

\begin{tabular}{|l|l|l|l|l|}
\hline F & M & Ph & Ce & $\begin{array}{l}\text { Tem- } \\
\text { peratura }\end{array}$ \\
\hline F2 & M1 & 8,34 Básico & $105,9 \mathrm{vs} / \mathrm{cm}$ & $21,9^{\circ}$ \\
\hline F2 & M2 & 8,12 Básico & $61,6 \mathrm{vs} / \mathrm{cm}$ & $21,7^{\circ}$ \\
\hline F2 & M3 & 8,55 L.Alc. & $97,0 \mathrm{vs} / \mathrm{cm}$ & $23,1^{\circ}$ \\
\hline F2 & M4 & 8,02 Básico & $107,1 \mathrm{vs} / \mathrm{cm}$ & $23,5^{\circ}$ \\
\hline
\end{tabular}


Tabla 3. Propiedades químicas del suelo de la comunidad Bajo de Afuera del cantón Montecristi.

Las propiedades físicas de un suelo son el resultado de la interacción que se origina entre las distintas fases del mismo (suelo, agua y aire) y la proporción en la que se encuentran cada una de estas. La condición física de un suelo determina su capacidad de sostenimiento, facilidad para la penetración de raíces, circulación del aire, capacidad de almacenamiento de agua, drenaje, retención de nutrientes, entre otros factores. En la Tabla $\mathrm{N}^{\circ} 4$ se presentan las propiedades físicas, que determinan que el suelo de la Comuna Bajo de Afuera es arcilloso.

\begin{tabular}{|l|l|l|l|}
\hline Celsius & Fahrenheit & Kelvin & Rankine \\
\hline $21,4^{\circ} \mathrm{C}$ & $\mathrm{F}=44,92^{\circ} \mathrm{F}$ & $\mathrm{K}^{\circ}=294,4^{\circ}$ & $\mathrm{R}=504,92^{\circ}$ \\
\hline $22,7^{\circ} \mathrm{C}$ & $\mathrm{F}=47,26^{\circ} \mathrm{F}$ & $\mathrm{K}^{\circ}=295,7^{\circ}$ & $\mathrm{R}=507,26^{\circ}$ \\
\hline $22,3^{\circ} \mathrm{C}$ & $\mathrm{F}=46,54^{\circ} \mathrm{F}$ & $\mathrm{K}^{\circ}=245,3^{\circ}$ & $\mathrm{R}=507,54^{\circ}$ \\
\hline $22,6^{\circ} \mathrm{C}$ & $\mathrm{F}=47,08^{\circ} \mathrm{F}$ & $\mathrm{K}^{\circ}=295,6^{\circ}$ & $\mathrm{R}=507,08^{\circ}$ \\
\hline $21,9^{\circ} \mathrm{C}$ & $\mathrm{F}=45,82^{\circ} \mathrm{F}$ & $\mathrm{K}^{\circ}=294,4^{\circ}$ & $\mathrm{R}=505,82^{\circ}$ \\
\hline $21,7^{\circ} \mathrm{C}$ & $\mathrm{F}=45,46^{\circ} \mathrm{F}$ & $\mathrm{K}^{\circ}=294,7^{\circ}$ & $\mathrm{R}=505,46^{\circ}$ \\
\hline $23,1^{\circ} \mathrm{C}$ & $\mathrm{F}=47,98^{\circ} \mathrm{F}$ & $\mathrm{K}^{\circ}=296,1^{\circ}$ & $\mathrm{R}=507,98^{\circ}$ \\
\hline $23,5^{\circ} \mathrm{C}$ & $\mathrm{F}=48,7^{\circ} \mathrm{F}$ & $\mathrm{K}^{\circ}=296,5^{\circ}$ & $\mathrm{R}=508,7^{\circ}$ \\
\hline
\end{tabular}

Tabla 4. Propiedades físicas del suelo de la comunidad Bajo de Afuera del cantón Montecristi.

De acuerdo a los resultados de la encuesta realizada podemos constatar que el $10 \%$ de los habitantes encuestados indicaron que si conocen el tipo de suelo con el que cuenta su comunidad, mientras que el 90\% indicaron que no. La información revela que no que no existe un conocimiento y no identifican el tipo de suelo de la comunidad, se observa Tabla \# 5 y el gráfico 2.

\begin{tabular}{|l|l|l|}
\hline Alternativa & Frecuencia & $\%$ \\
\hline $\mathrm{Si}$ & 5 & $10 \%$ \\
\hline No & 45 & $90 \%$ \\
\hline TOTAL & 50 & $100 \%$ \\
\hline
\end{tabular}

Tabla 5. Conocimiento del tipo de suelo por parte de la comunidad.
Gráfico 2. Conocimiento del tipo de suelo por parte

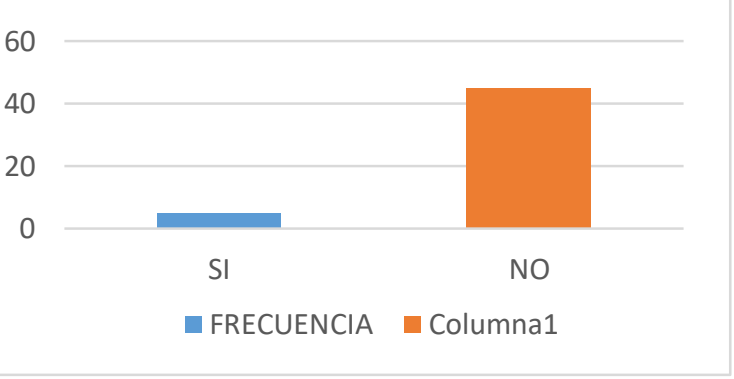

de la comunidad.

En cuanto a las actividades agrícolas a las que se dedican en la comunidad, el maíz es un cultivo muy importante desde su surgimiento, tanto para nuestra alimentación como para la alimentación animal. El maíz es el principal cultivo y renglón económico de los habitantes de los Bajos de Afuera del cantón Montecristi; sin embargo, sus producciones son insuficientes y económicamente insostenibles. A esta actividad solo se dedica un $50 \%$ de la población y se la realiza en la época de invierno.

Se pudo constatar que el $50 \%$ de los habitantes se dedican al cultivo de maíz, el $30 \%$ al criadero de chivos de castilla y el $20 \%$ al criadero de aves de corral. (Tabla \# 6). Por lo que se puede concluir que la mayor parte de la comunidad se dedica al cultivo de maíz y el animal de crianza es el chivo de casti1la, por el cual es renombrado nuestro sector ya que se realiza la Feria del Seco de Chivo, anualmente en el mes de noviembre.

\begin{tabular}{|l|l|l|}
\hline Actividad Agrícola & Frecuencia & $\%$ \\
\hline Cultivo de Maíz & 25 & $50 \%$ \\
\hline Criadero de aves de corral & 10 & $20 \%$ \\
\hline Criadero de chivos de castilla & 15 & $30 \%$ \\
\hline TOTAL & 50 & $100 \%$ \\
\hline
\end{tabular}

Tabla 6. Actividades agrícolas a las que se dedica la comunidad

En lo referente a las plagas que generalmente afectan los cultivos, se pudo determinar que el gusano cogollero es el principal causante de la perdida de cultivo de maíz en el Bajo de Afuera del cantón Montecristi. Para combatir la plaga que afectan el cultivo de maíz, se utiliza los siguientes insecticidas. La plaga del gusano cogollero es el principal causante de la perdida de cultivo de maíz en el Bajo de Afuera del cantón Montecristi. Para combatir la plaga que afectan el cultivo de maíz, se utiliza los 
siguientes insecticidas: Radiant y su ingrediente activo, es spinetoram, tabla 7 .

\begin{tabular}{|l|l|l|}
\hline Plagas & Frecuencia & $\%$ \\
\hline Gusano Cogollero & 35 & $70 \%$ \\
\hline Cinta Amarilla & 10 & $20 \%$ \\
\hline Quemazón & 15 & $30 \%$ \\
\hline Total & 50 & 100 \\
\hline
\end{tabular}

Tabla 7. Plagas que afectan los cultivos.

En cuanto a la pregunta realizada podemos constatar que el $20 \%$ de los habitantes encuestados utilizan fertilizantes Radiant, mientras que el $80 \%$ utilizan Endgusamyl, para el control de insectos y plagas, se observa en la tabla 8 .

\begin{tabular}{|l|l|l|}
\hline Fertilizantes & Frecuencia & $\%$ \\
\hline Radiant & 10 & $20 \%$ \\
\hline Endgusamyl & 40 & $80 \%$ \\
\hline Total & 50 & $100 \%$ \\
\hline
\end{tabular}

Tabla 8. Fertilizantes que utilizan para sus cultivos.

\section{Discusión}

La investigación se basa en la identificación del suelo de la comunidad Bajo de Afuera del cantón Montecristi, además de conocer su población, sus cultivos, y los principales problemas que se centran dentro de los mismos, como son las plagas que generalmente en la etapa de cultivos son los causantes de la pérdida del producto y por ende afecta a la economía de los agricultores. A través de la investigación se demuestra a los estudiantes de la carrera Agropecuaria la relación con las ciencias básicas como la química, física, biología y matemáticas.

El desarrollo de Proyectos Integradores de Saberes (PIS) en la Universidad Estatal de Manabí y en específico en la carrera agropecuaria es una actividad fundamental dentro del rediseño curricular y de gran desafío, porque implica la integración de diferentes ciencias del saber a través de un proyecto conjunto y coordinado, en este sentido se desarrolló esta propuesta, con los objetivos de desarrollar, fortalecer, motivar las capacidades cognoscitivas, psicomotrices y actitudinales en los estudiantes, a través del fortalecimiento de sus conocimientos en cuanto a la identificación de propiedades del suelo agrícola en la zona de planificación 4"(G.M. 00306, 2017 ).
Se concuerda con el autor (Senplades, 2015), en la que hace alusión al buen vivir, señala que se planifica, no se improvisa y la respuesta de Ecuador al reto de conseguirlo, se materializa en el Sistema Nacional Descentralizado de Planificación Participativa y concretamente en sus instrumentos. Los mismos aportan al desarrollo integral, contemplando el crecimiento en los ámbitos económicos y socioculturales, a través de la correcta localización de sus actividades y permitiendo así reducir los desequilibrios territoriales existentes.

En lo relativo a las propiedades de los suelos, determinan en gran medida, la capacidad de muchos de los usos a los que el hombre los sujeta. La condición física de un suelo, determina, la rigidez y la fuerza de sostenimiento, la facilidad para la penetración de las raíces, la aireación, la capacidad de drenaje y de almacenamiento de agua, la plasticidad, y la retención de nutrientes, se concuerda con (Herrera, 2017; InfoAgro, 2017; Intagri, 2017).

En cuanto a las actividades agrícolas a las que se dedican en la comunidad, refieren que el maíz es un cultivo muy importante y constituye un renglón económico de los habitantes de los Bajos de Afuera del cantón Montecristi, se concuerda con otros autores (Botta et al. 2007), en la que hacen referencia a este cultivo de corto, el cual puede desarrollarse a diferentes pisos altitudinales y en consecuencia se adapta a diversos ambientes, Ecuador.

Se concuerda con (Valarezo, Cañarte, Navarrete, \& Muñoz, 2010), en la que plantea que en Ecuador, el gusano cogollero causa grandes pérdidas económicas, en las que se puede reducir entre un 10 y $60 \%$ el rendimiento del maíz. En México otros autores hacen referencia a los daños causados por el gusano cogollero (Valdez, Benigno, Soto, Osuna, Báez \& Alonso 2012).

Es por ello que en el Proyecto Integrador de Saberes de la carrera Agropecuaria el estudiante tiene un espacio físico para realizar el estudio de los suelos y conocer las relaciones entre las diferentes materias integrando saberes donde se lleva a cabo las etapas de diagnóstico, diseño y evaluación, las cuales directamente utilizan los conocimientos básicos de las asignaturas que recibe el estudiante en el primer semestre. (Lógica Matemática, Algebra, Física, Química General, Biología Celular y Expresión Oral y Escrita).

A través de ésta investigación se fortalecen los conocimientos para lograr un cambio sistémico (de actitud) de las percepciones y criterios de conoci- 
miento, a través de lograr el desarrollo de un proyecto integrador de saberes, que articule los diferentes campos del saber impartidos de una manera natural y según como acontece realmente en la producción de los sistemas agropecuarios con el fin de fortificar las capacidades cognoscitivas, psicomotrices y actitudinales en los estudiantes de la carrera Agropecuaria, a través del estudio sobre el análisis de las propiedades químicas del suelo en la comunidad El Bajo de Afuera del cantón Montecristi provincia de Manabí

Los estudiantes llegan con conocimientos diferentes en los campos del saber, algunos con muchas debilidades y criterios equivocados sobre la carrera agropecuaria, como el hecho de pensar que esta carrera no tiene relación con las ciencias básicas como la química, física, biología y las matemáticas. Muchos tienen deficiencias serías en los conocimientos básicos de estas asignaturas. Los nuevos estudiantes no ven la relación de las ciencias exactas y las ciencias biológicas, menos comprenden sobre la importancia de la interpretación y presentación de resultados.

Con el desarrollo del proyecto integrador de saberes los estudiantes pueden integrar los diferentes conocimientos de las ciencias básicas que estudian en los primeros semestres y después los aplican en las ciencias agropecuarias de la especialidad, así como en las horas de vinculación con la sociedad que cumplen en el octavo semestre y las prácticas pre profesionales en el séptimo semestre.

La investigación se apoya en la Biología para conocer la estructura y formas de combatir las plagas, la Química para las diferentes transformaciones de las sustancias, la Física la utiliza para conocer las propiedades del suelo, los procesos físicos que ocurren en su superficie, y que son importantes en el sector agrícola para predecir los factores que intervienen en ecosistemas naturales y simulados como el crecimiento de las plantas. Además, las matemáticas con las cuales se obtienen los resultados estadísticos, tablas y gráficos de la investigación y la Expresión oral para comunicar de forma clara y precisa lo que se quiere transmitir.

Los resultados a obtener son estudiantes motivados, que hayan desarrollado sistémicamente su cambio de mentalidad, actitud y sensibilidad hacia la naturaleza, que tengan herramientas teórico-prácticas sólidos para interpretar los fenómenos, motivados en su inventiva e ingenio y hayan avanzado sus habilidades para expresar sus ideas apropiadamente, permitiendo el desarrollo integral de la persona, re- articulando la investigación, formación y vinculación. Aspectos que serán dados a través de las vinculaciones interdisciplinarias entre todas las asignaturas del saber impartidas en el primer semestre de la carrera Agropecuaria. (G.M.003-06, 2017)

\section{Conclusiones}

A través de la investigación los estudiantes de la carrera Agropecuaria desarrollan las capacidades cognoscitivas, psicomotrices y actitudinales integrando los saberes que le aportan las ciencias básicas, a través del estudio sobre "La identificación de propiedades del suelo agrícola en la Comuna Bajo de Afuera del cantón Montecristi”.

Los suelos con fines agrícolas de la parroquia son, ácidos y alcalinos y que su principal cultivo y renglón económico es el maíz; sin embargo, sus producciones son insuficientes y económicamente insostenibles, a esta actividad solo se dedica un $50 \%$ de la población y la realiza en la época de invierno y un $30 \%$ se dedica a la crianza de chivo de castilla, por el cual es renombrado el sector ya que se realiza la Feria del Seco de Chivo, anualmente en el mes de noviembre.

Las ciencias básicas tienen las potencialidades para con el estudio de los diferentes contenidos ir potenciando el desarrollo del aprendizaje de los estudiantes integrando los saberes con las ciencias agropecuarias y poder capacitar a la comunidad para lograr una mejor preparación de los agricultores de la zona en el uso del suelo y aplicación de fertilizantes.

\section{Bibliografía}

Botta, O., Pozzollo, M., Bomben, M., Tourn, E., Soza, H., Rosatto, A. Gili, J., Ressia, D., Rivero,

J., Vásquez, et al. (2007). "Aplicación del trafico controlado en la cosecha de maíz (zea mays 1.) efecto sobre rendimientos del cultivo y las propiedades físicas del suelo.," Agro-Ciencia. $\mathrm{V},(23), 1,7-16$.

Guía Metodológica Para la Elaboración del Proyecto Integrador de Saberes en la Universidad Estatal del sur de Manabí. (2017). Resolución número 003-06-(2017).

Herrera, J. (2017).Propiedades del suelo. Monografias Plus. Recuperado de 
https://m.monografias.com/trabajos65/propie dades-suelo/propiedades-suelo.shtml

InfoAgro. (2017). Composición quimica del suelo y su Ph. Recuperado de http://mexico.infoagro.com/composicionquimica-del-suelo-y-su-ph/

Intagri. (2017). Propiedades Físicas del Suelo y el Crecimiento de las Plantas. Recuperado de https://www.intagri.com/articulos/suelos/prop iedades-fisicas-del-suelo-y-el-crecimiento-delasplan-

tas\#: :text=El\%20di\%C3\%A1metro\%20de\% 20las\%20part\%C3\%ADculas,el\%20uso\%20d e\%20implementos\%20agr\%C3\%ADcolas.

Tamhane, R., Motiramani, D., Bali, Y. y Donahue R. (1986). Suelos: su química y fertilidad en zonas tropicales. Editorial Diana Técnico. México D.F. 483 pp. United States Department of Agriculture.

Valarezo, O., Cañarte, E., Navarrete, B., \& Muñoz, X. (2010). Manejo integrado de las principales plagas del Maíz. INIAP, Plegable Divulgativo, 389, 1-10.

Valdez, T. (2014). Pis Proyecto Integrador de Saberes. Recuperado de: http://nivelacionsiglo21.blogspot.com/

Valdez, J., Benigno, S., Osuna, F. Báez, T., Alonso, M. (2012). Modelos de predicción fenológica para maíz blanco (Zea mays L.) Y gusano cogollero (Spodoptera frugiperda J. E. Smith) Agrociencia, (46), 4, 399-410. Recuperado de:

https://www.redalyc.org/pdf/302/3022311000 7.pdf 